\title{
Water and Oil Repellent Finishing of Textiles by UV Curing: Evaluation of the Influence of Scaled-Up Process Parameters
}

\author{
Franco Ferrero ${ }^{1, *}$, Monica Periolatto ${ }^{1}$ and Lorenzo Tempestini ${ }^{2}$ \\ 1 Department of Applied Science and Technology, Politecnico di Torino, Corso Duca degli Abruzzi 24, \\ 10129 Torino, Italy; monica.periolatto@polito.it \\ 2 Jersey Mode Spa, Via delle Fonti 384, 59100 Prato, Italy; lorenzo.tempestini@jerseymode.it \\ * Correspondence: franco.ferrero@polito.it; Tel.: +39-011-090-4630 \\ Academic Editor: Mahbubul Hassan
}

Received: 30 November 2016; Accepted: 20 April 2017; Published: 26 April 2017

\begin{abstract}
In this work, various textile fabrics were coated with silicone and fluorocarbon-based resins by photo-curing using ultraviolet irradiation. A great number of large fabric samples were impregnated by padding with commercial finishing agents and then irradiated in air with a high power, semi-industrial UV source. The add-on of various finishing agents was kept low to reduce the treatment cost. White and dyed samples of different textile composition were treated and evaluated in terms of conferred repellency, yellowing, or color changes. Most relevant process parameters were investigated, utilizing the thermal process normally adopted at industrial level as reference. The results were statistically evaluated by ANOVA using Minitab 16 software, in order to identify the most influential parameters and to evaluate the real possibility of replacing the thermal treatment with UV curing.
\end{abstract}

Keywords: cotton fabric; viscose fabric; coating; UV curing; water repellency; oil repellency

\section{Introduction}

Water and oil repellency are among the most common functional properties necessary for protective clothing. These properties can be conferred by the modification of the surface energy of textile fibers, possibly confined to a thin surface layer, so that the bulk properties of the fabric, such as mechanical strength, flexibility, breathability, and softness remain uncompromised [1,2].

The modification routes should meet the criteria of green chemistry as much as possible. In particular, the use of harmless solvents and non-toxic chemicals should be a characteristic of the process. This principle, in agreement with the ecological orientation of industrial textile chemistry, limits in a significant way the number of viable strategies and reactions. Among them, photografting by UV light combines the inherent benefits of photochemical initiation, namely a fast, versatile, efficient process and the advantage of a surface grafting that does not modify the bulk properties nor degrade the fiber structure.

Grafting has advantages over other modification methods in several ways, including easy and controllable introduction of a high density of graft chains with exact localization of graft sites on the fabric surface. Furthermore, covalent attachment of graft chains onto a textile surface ensures the long-term chemical stability of introduced chains. This is in contrast to what may be aspected for physical coating on polymer chains obtained by thermal treatment. The use of UV irradiation appears to be an excellent method for surface grafting of functional monomers on textile fibers. Photochemically-produced triplet states of carbonyl compounds of a suitable photoinitiator can abstract hydrogen atoms from almost all polymeric materials (such as textile fibers) so that graft polymerization 
may be initiated [3,4]. Moreover, when UV irradiation is applied, high concentrations of active species can be produced locally at the interface between a textile fiber and the monomer solution containing the photoinitiator, with the consequence of a high yield for the process. The mechanism of UV-initiated polymeric grafting and the grafting process are shown in Figures 1 and 2, respectively.

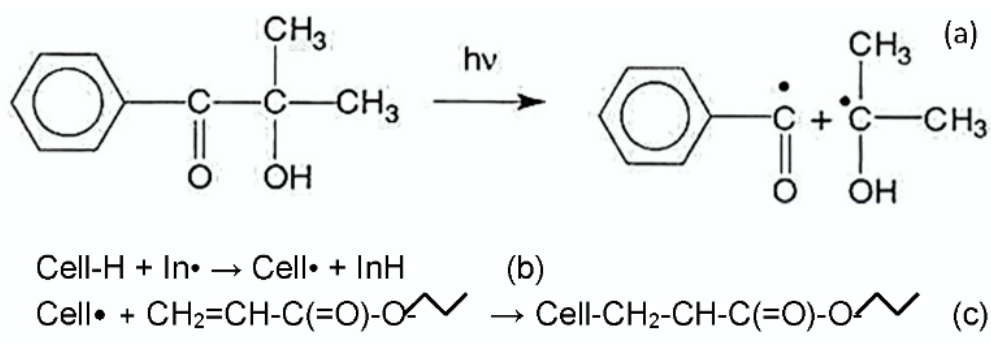

Figure 1. UV grafting mechanism: (a) Photoinitiator (Darocur 1173) fragmentation; (b) H abstraction from cellulose backbone; (c) grafting of acrylic oligomer on cellulose.

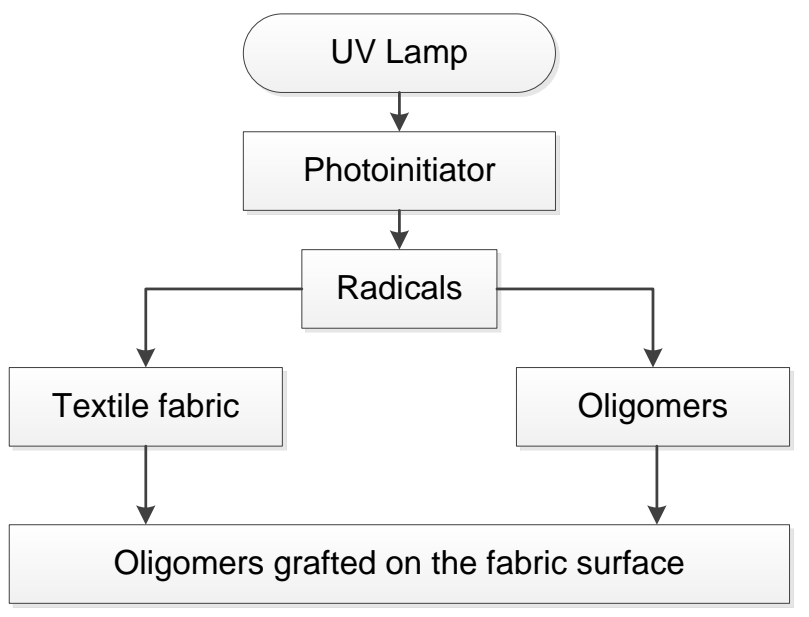

Figure 2. UV grafting process on fabric.

Besides the well-known advantages of UV curing (space saving, easy installation of equipment, reduced waste, high speed of treatment) [5], this process consumes only about $25 \%$ of the energy required by a conventional thermal-based system using a fuel-fired oven [6], since it is carried out at low temperature. Therefore, the functionalization of a cotton surface by UV-cured coatings has been proposed to obtain various finishing effects onto fabrics such as color fastness in pigment printing [7], antimicrobial activity [8], flame retardancy [9,10], and finally water and oil repellency [11,12].

Polysiloxanes are widely used for textile finishing to impart softness, crease resistance, and water repellency. A typical molecular structure of silicone polyether oligomer containing reactive acrylic groups for thermal or UV curing is shown in Figure 3.

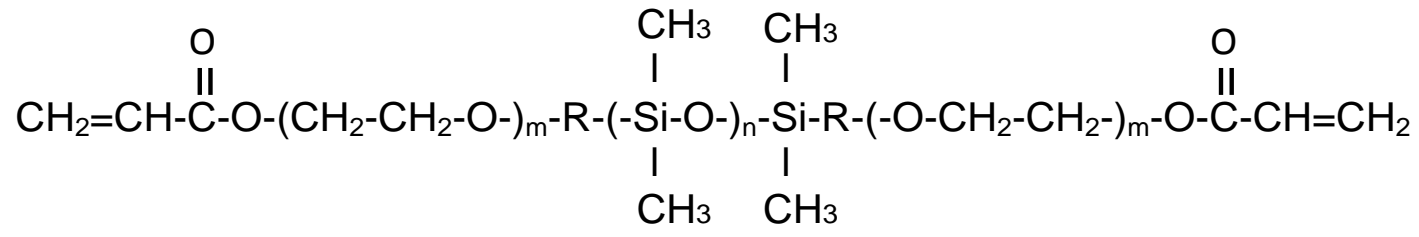

Figure 3. Molecular structure of diacrylated silicone polyether oligomer.

The application by UV grafting of polyhedral oligomeric silsesquioxanes (POSSs) and polysilazane (KION 20) to cotton fabrics to confer water repellency has recently been reported [13]. This was clearly 
achieved by the treatment with both silicon compounds showing water contact angles higher than $90^{\circ}$ on the as prepared samples, but with total loss of water repellency after aging of those treated with POSS.

However, silicon compounds are unable to confer oil repellency, hence the production and application of different types of fluorochemicals for textile finishing has often been reported [14]. These consist of perfluorinated carbon chains which impart, at the same time, water and oil repellency to the fiber surface when incorporated into a polymer backbone with perfluoro groups as side chains [15].

The currently used fluorochemicals are based on C6 carbon chains, which have replaced the C8 fluorocarbons that can release perfluorooctanesulfonate (PFOS) and perfluorooctanoic acid (PFOA), higly hazardous and toxic substances. In fact, in 2009 Huntsman launched Dupont $^{\mathrm{TM}}$ (Dupont, Wilmington, DE, USA) products under the name Oleophobol ${ }^{\circledR} \mathrm{CP}$ (Oleophobol, Huntsman, Switzerland) based on short chain molecules that cannot break down into PFOA in the environment and provide a step-change reduction in trace impurities below the limit of detection. However, C6-based fluorocarbons show repellency rating and washing durability much lower than C8-based ones, hence cross-linkers, such as maleic acid, were introduced to enhance their performance and durability [16].

A typical molecular structure of these short-chain perfluorinated oligomers containing reactive acrylic groups for thermal or UV curing is reported in Figure 4.

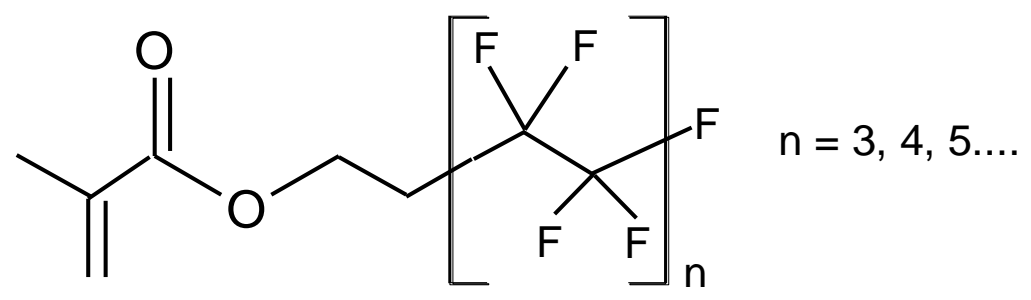

Figure 4. Molecular structure of perfluoro-acrylic oligomers.

Fluorochemical finishings are commercially available as water emulsions and are applied to fabrics by the pad-dry-cure method, with a thermal curing step at $150-175{ }^{\circ} \mathrm{C}$ in a hot flue for some minutes [17-20]. As an alternative, the UV grafting of perfluoro-alkyl-polyacrylate resins as Oleophobol $\mathrm{CP}-\mathrm{C}$ has been tested on cotton fabrics in a previous laboratory work, where the effectiveness of this approach has been compared with that obtained by thermal polymerization [2]. Yields after UV curing are quite similar, while strong water and oil repellency conferred by photografting was confirmed by static and dynamic contact angle measurements. The water repellency was practically unaffected by repeated washings regardless of curing time and finish add-on. Water vapor transmission rates showed that both the thermal and the UV curing finish treatments do not reduce the breathability of the original cotton. The laboratory experiments were carried out on dried samples of small surface by UV grafting under nitrogen flux, using a low power apparatus, as recommended to maximize the yield of radical curing. In conclusion, the laboratory scale application of water and oil repellent finishing on cotton fibers by UV grafting of silicone or fluorocarbon-based resins, with the optimization of process parameters, followed by a deep characterization of treated samples, confirmed the effectiveness of the treatment.

The aim of the present study has been a semi-industrial scale-up of the process. The scaling-up is one of the aspects of photografting that requires more efforts to optimize the different parameters affecting the final efficiency of the process, such as the chemistry of the reagents, type and concentration of the photoinitiator, and UV dose and its delivery, especially in a continuous roll-to-roll process. A great number of fabric samples larger than those used in laboratory experiments has been padded with commercial finishing agents and then irradiated with a high power, semi-industrial UV lamp. The treatment has been performed in air to avoid the inert gas consumption. Finishing agent add-ons have been significantly kept low in order to hold down the finishing cost. White and dyed samples 
of different textile composition have been treated and evaluated in terms of conferred repellency, yellowing, or color changes.

Most relevant process parameters have been investigated, considering the thermal process normally adopted at the industrial level as reference. Results have been so statistically evaluated to point out the most influencing parameters and the real possibility to replace at industrial scale the thermal treatment with UV. The statistical treatment was performed using Minitab 16 software and the results are reported in Supplementary Materials, where Table S1 gives the meaning of the Minitab codes, while the Figures from S1 to S10 show the statistical plots.

\section{Materials and Methods}

In this work, three different knitted fabrics were used:

- "Punto Milano" (PM) dark grey heavy fabric, $360 \mathrm{~g} / \mathrm{m}^{2}$, composed by $70 \%$ viscose, $25 \%$ nylon and $5 \%$ elastan;

- $\quad$ white pure cotton (COT), $210 \mathrm{~g} / \mathrm{m}^{2}$;

- white light viscose (VIS), $130 \mathrm{~g} / \mathrm{m}^{2}$, composed by $94 \%$ viscose and $6 \%$ elastan.

Oleophobol CP-S (Oleophobol, Huntsman, Switzerland) and Pymasil E-MC (Pymasil, Pymag, Spain) were applied as finishing agents. Both are commercial products in form of aqueous emulsions stabilized with surfactants, with about $20 \mathrm{wt} \%$ concentration of fluoro-acrylic or silicone-acrylic oligomers, for Oleophobol and Pymasil, respectively. They are already applied at an industrial level by thermal process to confer stain resistance (Oleophobol) and soft hand (Pymasil).

A great number (180) of fabric samples $\left(40 \times 20 \mathrm{~cm}^{2}\right)$ were padded with Oleophobol or Pymasil solutions ( $50 \mathrm{~g} / \mathrm{L}, 35 \mathrm{~g} / \mathrm{L}$, and $20 \mathrm{~g} / \mathrm{L}$ for the first; $40 \mathrm{~g} / \mathrm{L}, 25 \mathrm{~g} / \mathrm{L}$, and $10 \mathrm{~g} / \mathrm{L}$ for the second), while for UV curing $4 \mathrm{wt} \%$ of Darocur 1173 (Ciba) finishing agent was added as photoinitiator. The samples were dried at $80{ }^{\circ} \mathrm{C}$ for $60 \mathrm{~s}$, and finally irradiated in air by a static mercury discharge UV lamp providing three tunable irradiances: $146 \mathrm{~mW} / \mathrm{cm}^{2}, 85 \mathrm{~mW} / \mathrm{cm}^{2}$, or $67 \mathrm{~mW} / \mathrm{cm}^{2}$. Samples were exposed to the radiation on one side for a time of $60 \mathrm{~s}$. The photoinitiator concentration was assessed after previous tests aimed to obtain the maximum grafting yield of the oligomers in the adopted irradiation conditions without stickiness on the final fabric. It is worth to noting that, by UV irradiation in air rather than in nitrogen, the radical reactions are slackened by oxygen interference, hence a higher photoinitiator concentration is needed to speed up the initiation step. In fact, in the previous laboratory experiments of irradiation in inert atmosphere a $2 \mathrm{wt} \%$ photoinitiator concentration on finishing agent was enough to achieve satisfactory grafting yields [2].

The flow diagram of the UV grafting process is schematically depicted in Figure 5. The thermal curing on reference samples was carried out following the same diagram without photoinitiator addition, while the irradiation step was substituted by thermal treatment in a hot flue at $150{ }^{\circ} \mathrm{C}$ for $5 \mathrm{~min}$. Three samples for each process were prepared for a statistical treatment of data.

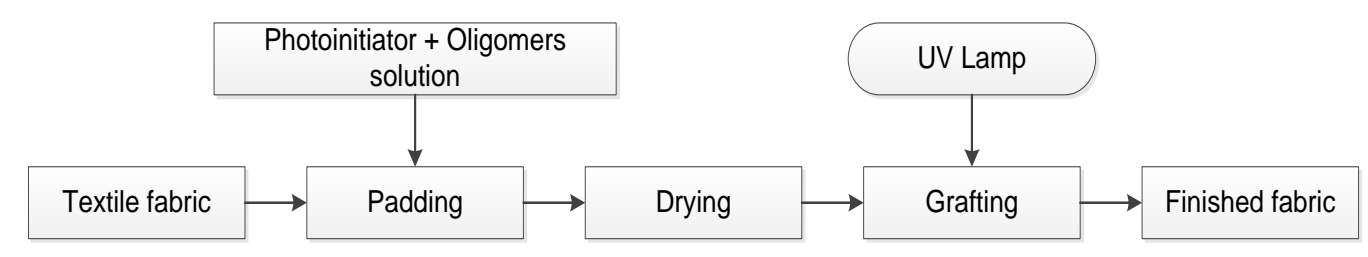

Figure 5. Flow diagram of the UV grafting process.

Water and oil absorbency were tested by measuring the time it takes a drop of liquid placed on the fabric surface to be completely absorbed into the fabric, according to AATCC/ASTM Test Method TS-018 [21]. The contact angles (CA) were evaluated by a CA tensiometer DSA20E (Krüss $\mathrm{GmbH}$, Hamburg, Germany) equipped with a software for drop profile fitting (Krüss GmbH, Hamburg, 
Germany). The test liquids used were HPLC-grade water and paraffin oil (Sigma Aldrich, Milano, Italy) with surface tension of $72 \mathrm{mN} / \mathrm{m}$ and $31.5 \mathrm{mN} / \mathrm{m}$, respectively. The contact angles considered were the average of at least five measurements for each sample with a standard deviation of about $2-3 \%$.

Color measurements were carried out by a Datacolor 110R reflectance spectrophotometer (Datacolor AG Europe, Dietlikon, Switzerland), which enables the determination of color differences $\Delta E$ between a sample and a reference through the formula:

$$
\Delta E=\sqrt{(\Delta L)^{2}+(\Delta C)^{2}+(\Delta h)^{2}}
$$

where $\Delta L, \Delta C$, and $\Delta h$ are the differences of lightness, chroma, and hue respectively. For color tolerance evaluation in textile industry, the CMC (Color Measurement Committee) system is currently used [22]. An ellipsoidal volume around the position of the color reference in the color space is calculated as function of $\Delta L, \Delta C$, and $\Delta h$. If a sample fall inside the ellipsoid is deemed acceptable, otherwise it is rejected. According to this system, $\Delta E=1$ is assumed as limiting value under which samples can be considered to have the same color as the reference.

All measurements were then statistically evaluated by ANOVA with Minitab 16 software (Minitab Inc., State College, PA, USA), in order to point out the most influencing parameters. The plots obtained by this approach are reported in the Supplementary Material (SM).

\section{Results and Discussion}

The study about water and oil repellent finishing has been performed on the three fabrics of different composition and color. The main parameters considered and varied, besides the substrate, were the finishing agent and the application process. Irradiance and concentration of the finishing agent in the impregnation solution, which are directly related to the final add-on, were the process parameters further investigated.

Obtained results were evaluated in terms of absorbency of water or oil drops on the treated surface, contact angles (CA), and color measurements.

\subsection{Absorbency of Water and Oil Drops}

Absorption times of water or paraffin oil drops, with a volume of about $10 \mu \mathrm{L}$, were evaluated on PM and COT samples treated by Pymasil or Oleophobol. If the drop was not absorbed after $120 \mathrm{~min}$, the test was stopped and the substrate was considered completely repellent toward the considered liquid. Results and process parameters are summarized in Table 1.

Table 1. Absorbency of drops on treated samples.

\begin{tabular}{ccccccc}
\hline Method & Fabric & Finishing Agent & Concentration $\mathbf{( g / L )}$ & Irradiance $\left(\mathbf{m W} / \mathbf{c m}^{2}\right)$ & Water $(\mathbf{m i n})$ & Oil $(\mathbf{m i n})$ \\
\hline Thermal & PM & Oleophobol & 50 & - & $>120$ & $>120$ \\
Thermal & PM & Pymasil & 40 & - & 49 & 1 \\
Thermal & COT & Oleophobol & 50 & - & $>120$ & $>120$ \\
Thermal & COT & Pymasil & 40 & - & 34 & 1 \\
UV & PM & Oleophobol & 50 & 67 & $>120$ & $>120$ \\
UV & PM & Oleophobol & 20 & 67 & $>120$ & $>120$ \\
UV & PM & Oleophobol & 20 & 146 & $>120$ & $>120$ \\
UV & PM & Pymasil & 10 & 67 & 90 & 2 \\
UV & COT & Oleophobol & 50 & 67 & $>120$ & $>120$ \\
UV & COT & Oleophobol & 50 & 146 & $>120$ & $>120$ \\
UV & COT & Oleophobol & 20 & 67 & $>120$ & $>120$ \\
UV & COT & Oleophobol & 20 & 146 & 80 & $>120$ \\
UV & COT & Pymasil & 40 & 67 & & 1 \\
\hline
\end{tabular}

The high efficiency of Oleophobol, regardless the application method and the process parameters chosen for the finishing, is clear. Oleophobol can act both as water and oil repellent finishing, as confirmed by absorption times of both drops higher than $120 \mathrm{~min}$. 
Considering Pymasil, the repellent effect was observed only on water drops, as expected, due to the absence of fluorinated chains on the oligomer molecules, which are responsible for the oil repellency. Silicone chains can confer good water repellency: The higher absorption time for a water drop was in fact 80-90 min. However, better results were obtained with Oleophobol due to its greater efficacy as repellent agent, while Pymasil is not a specific water repellent finishing, but a softener that can confer some water repellency thanks to the silicone component of its formulation.

The tests with Pymasil were useful to put in evidence the influence of the finishing method: better results were obtained by UV radiation, rather than thermal process. When the finishing agent was applied by UV curing, the water drop stays unadsorbed on the treated surface for more than twice as long as the thermal treated samples. This can be ascribed to a stronger bond between the finishing agent and the fibers, due to grafting reactions induced by ultraviolet radiation.

\subsection{Contact Angle Measurements}

The CA measured on all treated samples clearly showed the effect of the finishing agent: Values near or higher than $150^{\circ}$ were always obtained, with both water and oil, in the case of fluorocarbon finishing, while similar water CA were measured on samples treated by the silicone-based product, regardless the fabric considered. These values, typical of surfaces with super-repellency showing the so-called "lotus effect", have to be compared with a $0^{\circ} \mathrm{CA}$ measured on untreated samples with both water and oil, due to instantaneous absorption of the drop. Nevertheless, the influence of the process parameters was quite different considering the two finishing agents.

\subsubsection{Oleophobol}

The results of water and oil CA on PM fabric vs. Oleophobol concentrations for each UV irradiance value are plotted in Figure 6. The averaged determinations on triplicate samples for each process are shown together with the error bars based on standard deviation. However, this type of graphical representation can show the dependence of CA on two process parameters only for a single substrate. Instead, to jointly evaluate the influence of fabric type, finishing concentration, and UV irradiance, the Matrix Plot was drawn by Minitab 16 and depicted in Supplementary Material (Figure S1). A matrix plot is a graph enabling assessment of the relationship among several pairs of variables at the same time.

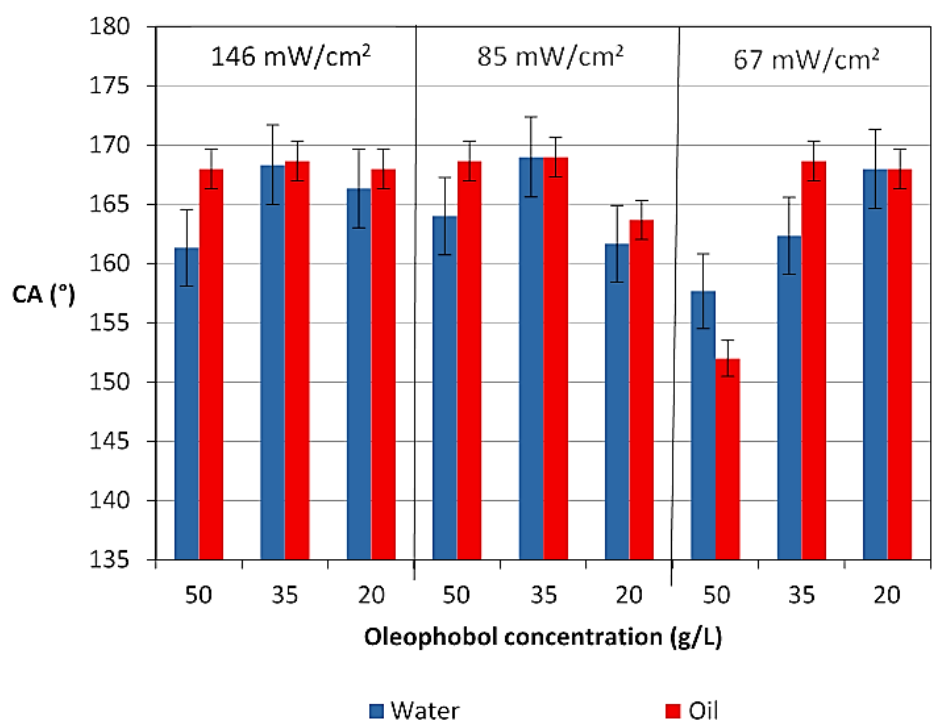

Figure 6. Water and oil CA on PM fabric vs. Oleophobol concentrations for each UV irradiance.

The substrate was found to be the more influencing parameter, in particular on water CA. This result was confirmed even by the analysis of variance (ANOVA). The best results were obtained on 
VIS while the lowest repellency was conferred to PM fabric. The chemical composition of these textiles is quite similar, so the different behavior could be due to the differences in color, texture, and weight. On the contrary, the substrate unaffected the conferred oil repellency, which slightly increased applying the product at higher concentration. Of low importance was the influence of product concentration and irradiance: A slight increase in oil and water repellency was found with the lower concentration, while the increase of the contact angles due to an increased lamp irradiance was negligible.

The majority of CA measurements was arranged at higher values: On a total of 81 measurements, just 17 were lower than $160^{\circ}$ ( 10 for water and 7 for oil), but never lower than $150^{\circ}$, confirming the super oil and water repellency conferred.

Then, the statistical analysis was further performed drawing an Interaction Plot referred to water repellency (Figure S2 in Supplementary Material). This plot enables visualization of possible interactions when the effect of one factor depends on the level of an another factor. The means for the levels of one factor are displayed on the $x$-axis, while a separate line for each level of another factor is drawn. Parallel lines in an interaction plot indicate no interaction, while the greater the difference in slope between the lines, the higher the degree of interaction. However, the interaction plot does not alert if the interaction is statistically significant.

In Figure S2, the worst behavior was shown by PM fabric, but this was improved lowering the Oleophobol concentration or increasing the irradiance. Lowering the irradiance, CA on both COT and PM decrease, while on VIS it is the contrary. In general, the best results were obtained by applying a medium product concentration on COT under medium-high irradiance.

For what concerns the Interaction Plot for oil repellency (Figure S3 in Supplementary Material), similar results were found: medium product concentration was confirmed as optimal, but the effect of the irradiance on the "difficult" samples, that is PM with higher product concentration, was more influent with respect to water repellency.

On the other hand, a Main Effects Plot displays the means for each group within a categorical variable. A line connects the points for each variable. When the line is horizontal (parallel to the $x$-axis), there is no main effect present and the response mean is the same across all factor levels. When the line is not horizontal, there is a main effect present and the response mean is not the same across all factor levels. The steeper the slope of the line, the greater the magnitude of the main effect.

Although this plot can be used to display the effects, the appropriate ANOVA test must be performed and the statistical significance of the effects evaluated.

The Main effect plots for CA with both water and oil (Figures S4 and S5, respectively in Supplementary Materials) show that the optimal conditions to obtain the best results in terms of both water and oil repellency on white COT fabric is the treatment with medium product concentration and irradiance.

\subsubsection{Pymasil}

For Pymasil-treated samples, only the water contact angle was considered for discussion since oil repellency was not conferred by such finishing agent. Water CA measured on Pymasil-treated samples showed a wider dispersion compared to Oleophobol. In particular, of 81 values, 16 are lower than $160^{\circ}$ and 3 are lower than $150^{\circ}$, which is considered the lower limit for superhydrophobicity.

ANOVA analysis revealed the type of substrate as the most influencing parameter, while the effect of product concentration and irradiance were negligible. The effect, as reported in Interaction Plot and Main effect plot (Figures S6 and S7, respectively in Supplementary Materials) was opposite compared to Oleophobol results: Higher water repellency was in fact obtained on PM while VIS gave the worst results. In detail, PM and VIS show opposite behaviors: On the first, higher CA values are obtained with lower product concentration and the effect of the irradiance is negligible, while on VIS the best results are achieved with higher product concentration and higher irradiance. 


\subsubsection{Thermal vs. UV Curing}

Considering the drop absorption times, no substantial differences ascribable to the finishing process can be noted for Oleophobol. On the other hand, comparing the results on Pymasil-treated samples, UV curing improved the water absorption more than twice with respect to thermal curing.

Considering CA values on each fabric, the comparison between the results with the highest concentration of Oleophobol is reported in Figure 7. It is evident that UV irradiation on PM under medium and higher irradiance improves both water and oil repellency of treated fabrics compared to thermal process, while on VIS the results are improved even at lower irradiance. Instead, thermal and UV curing yield practically the same results on COT.

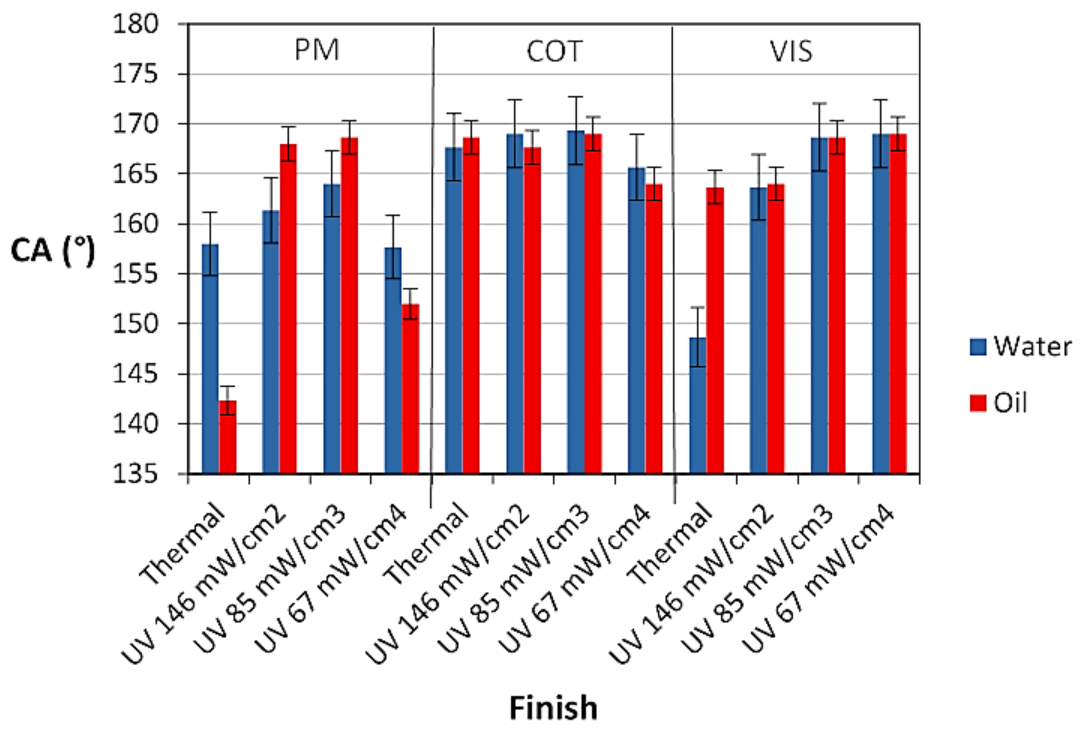

Figure 7. Water and oil CA on each fabric vs. finish type with $50 \mathrm{~g} / \mathrm{L}$ Oleophobol concentration.

\subsection{Color Evaluation}

Measurements of $\Delta E$ on differently treated samples showed that the effect of both yellowing or color changes are minimal with respect to untreated samples considered as references. In fact, in the Marginal Plot reported in Figure S8 in Supplementary Materials, a Gaussian distribution of $\Delta E$ values was observed for all 180 measurements, with most of the samples between 0.7 and 1.5 and only one sample with the greatest value of 3.6. Moreover, the color difference was mainly due to changes in yellowness.

The evaluation of Main effect plot (Figure S9 in Supplementary Materials) highlighted the type of fabric as the more sensitive parameter: On PM negligible color differences were measured regardless the process parameters, while a great influence of the curing method was revealed on VIS. This can be due to the different texture of the samples, as well as their color.

The thermal curing gave lower yellowing on VIS, while on PM and COT best results were obtained by UV. Moreover, the substrate color was more affected by Pymasil rather than Oleophobol. Finally, the influence of the product concentration was clear on COT fabrics, due to the higher absorption properties of the same.

By coupling the results reported in the Main effect plot (Figure S9) and Interaction Plot (Figure S10 in Supplementary Materials), something about the influence of the different process parameters can be deduced.

\subsubsection{Finishing Process}

The Main effect plot shows a little difference between the two methods, thermal or UV, with respect to the sample color. Samples treated by thermal process seem to keep the final color more 
similar to the original, if compared with UV treated samples. However, a lower number of samples were finished by thermal process rather than by UV; the color difference measurements carried out on thermal treated samples were homogeneously dispersed between 0.13 and 3.28 values. It means that the thermal method does not ensure a lower impact on the fabric appearance with respect to UV process.

The Interaction Plot, in fact, shows how better results related to thermal treatment are obtained with Oleophobol, but it is the finishing product giving best results at all. The main effect was found related to the substrate: on the dark PM both treatments can be considered similar, while on white COT the thermal process causes a higher color difference, mainly due to yellowing. On VIS there is a stronger effect of the radiation, due to the thin fiber diameter and larger opening of the fabric texture.

\subsubsection{Fabric Type}

The fabric type was the most influencing parameter on color differences due to the finishing process. A certain effect was revealed on COT and VIS, while on PM the color differences were negligible. It can be due to the fabric color (black is less sensitive) or grammage, which is higher for PM. Moreover, color differences measured on PM are less dispersed and close to the value 1, while on COT and VIS the values are more dispersed. For VIS, values lower than 1 were registered on all the thermal treated samples.

From the Interaction Plot, besides the different behavior due to the finishing method, no influence was evidenced by the finishing product. Moreover, considering the UV method, it can be noted that the influence of the different irradiance was quite ineffective on PM and COT, while for VIS the color degradation was higher for higher UV irradiance.

\subsubsection{Finishing Product and Concentration}

From the Main effect plot it is clear that the color differences are lower on Oleophobol compared to Pymasil. It was true also if the products were applied by thermal process. The dispersion of values was similar but for Pymasil the values were generally higher. Moreover for Pymasil there was also some influence of the product add-on, with a color difference higher if the add-on was higher. This parameter was unaffecting for Oleophobol.

The concentration of the finishing agent was the less influencing parameter on the final samples. As expected, best results were found with lower product concentrations, while the final color was affected increasing this concentration. As previously, it was not confirmed for Oleophobol, that was unaffected by the product concentration.

\subsubsection{Irradiance}

The best results were obtained on samples treated with the higher irradiance, improved even with respect to those thermally treated. The worst results, that is the most evident color difference after the treatment, were obtained on samples radiated with the lower UV irradiance. The most important effect was revealed on VIS fabric.

\subsection{Aging Tests}

The stability upon aging of the photografting treatment was checked. Resistance to aging was tested by measuring the contact angle on $20 \mathrm{~g} / \mathrm{L}$ Oleophobol-treated samples, two years after the surface treatment. The samples were stored in the laboratory at room temperature in the dark. The comparison of CA with water and oil between freshly prepared samples and aged samples is reported in Figure 8 for all the fabrics and irradiance values.

The aged samples were still found hydrophobic, with an average $15 \%$ reduction of the water contact angle regardless the irradiance. Moreover, the aged samples were still found oil repellent, but an average $40 \%$ reduction of the oil contact angles was measured. The worst results were obtained on VIS, while on COT fabrics the decrease was lower. The partial loss of oil repellency can be due 
to a rearrangement and orientation toward the inner part of the fibers of the surface fluorine chains, occurred during this time and mainly ascribable to some moisture content [23].

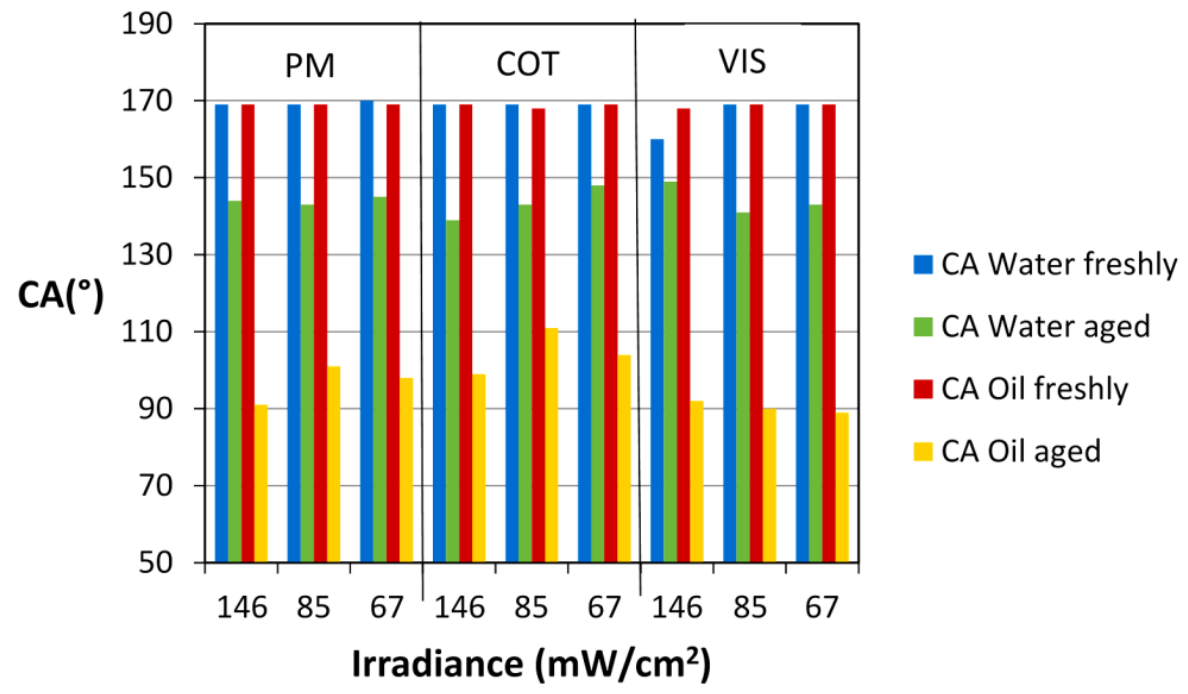

Figure 8. Water and oil CA on each fabric vs. irradiance. Comparison between fresly prepared and aged samples (two years) UV treated with $20 \mathrm{~g} / \mathrm{L}$ Oleophobol concentration.

\section{Conclusions}

In this work, various textile fabrics were coated with silicone and fluorocarbon-based resins by photo-curing using ultraviolet irradiation. A semi-industrial scale-up of the process was carried out: A great number (about 180) of large fabric samples were padded with Oleophobol or Pymasil finishing agents and then were irradiated in air by UV light with tunable irradiance. Samples were exposed to radiation on one side for $60 \mathrm{~s}$. The add-on of various finishing agents was kept low to reduce the treatment cost. White and dyed samples of different textile composition were treated and evaluated in terms of conferred repellency, yellowing, or color changes. The most relevant process parameters were investigated, considering the thermal process normally adopted at industrial level as reference. The results were statistically evaluated by ANOVA with Minitab 16 software, to point out the most influencing parameters and the real possibility of replacing the thermal treatment with UV.

Water and oil drop absorption times higher than 120 min were found on all Oleophobol-treated samples, showing that Oleophobol works very well as oil and water repellent agent for textiles. UV process was revealed to work better than the thermal one, in fact higher water and oil contact angles were obtained with a lower amount ( $1 \%$ o.w.f.) of finishing agent. Considering the UV process, best results were yielded by white fabrics, rather than dyed, and medium values of both irradiance and product concentration, taking into account both contact angles and color reflectance evaluations. Finally, contact angle measurements carried out on long aging time samples (two years) UV treated with Oleophobol showed only a low hydrophobicity decrease compared to fresh samples, while the loss of oil repellency was higher. Nevertheless, aged samples, particularly cotton, can still be considered oil repellent.

In conclusion, UV curing appears to be a valid alternative to the finishing thermal process actually applied at industrial level. By UV curing, in fact, same or even better water and oil repellency than thermal process were obtained, applying a low amount of finishing agent. It enables the better maintenance of textile properties coupled with the low process temperature, with a clear economic advantage.

Among UV treated samples, the type and color of treated fabrics had great influence on the obtained results, in particular for Pymasil; the best results were obtained by lowering the product concentration, while a negligible effect was due to different irradiance values. Finally, UV light caused 
lower yellowing compared to thermal treatment, with appreciable values just on viscose at higher radiation doses, due to the lightness of the fabric.

Obtained results were considered encouraging and can open the way for a real application of the UV process to industrial field.

Supplementary Materials: The following are available online at http://www.mdpi.com/2079-6412/7/5/60/s1. Table S1: Correspondence between Minitab codes and process parameters for the plots of Figures from S1 to S8. Figure S1: Matrix Plot on average values of water and oil CA on Oleophobol-treated samples by UV radiation, Figure S2: Interaction Plot for water CA on Oleophobol-treated samples, Figure S3: Interaction Plot for oil CA on Oleophobol-treated samples, Figure S4: Main effect plot for water CA on Oleophobol-treated samples, Figure S5: Main effect plot for oil CA on Oleophobol-treated samples, Figure S6: Interaction plot for water CA on Pymasil-treated samples, Figure S7: Main effect plot for water CA on Pymasil-treated samples, Figure S8: Marginal Plot for $\Delta E$ Datacolor measurements, Figure S9: Main Effects Plot for Datacolor measurements, Figure S10: Interaction Plot for Datacolor measurements.

Acknowledgments: Jersey Mode Spa is gratefully acknowledged for the financial and technical support.

Author Contributions: Monica Periolatto and Lorenzo Tempestini conceived and designed the experiments; Monica Periolatto performed the experiments and statistical analysis of the results; Monica Periolatto and Franco Ferrero analyzed the data and wrote the paper.

Conflicts of Interest: The authors declare no conflict of interest.

\section{References}

1. Bongiovanni, R.; Zeno, E.; Pollicino, A.; Serafini, P.M.; Tonelli, C. UV-light induced grafting of fluorinated monomer onto cellulose sheets. Cellulose 2011, 18, 117-126. [CrossRef]

2. Ferrero, F.; Periolatto, M.; Udrescu, C. Water and oil-repellent coatings of perfluoro-polyacrylate resins on cotton fibers: UV curing in comparison with thermal polymerization. Fiber. Polym. 2012, 13, 191-198. [CrossRef]

3. Roy, D.; Semsarilar, M.; Guthrie, J.T.; Perrier, S. Cellulose modification by polymer grafting: A review. Chem. Soc. Rev. 2009, 38, 2046-2064. [CrossRef] [PubMed]

4. Bongiovanni, R.; Chiappone, A.; Zeno, E. UV-Grafting: A powerful tool for cellulose surface modification. In Cellulose-Based Grafted Copolymers: Structure and Chemistry; Thakur, V.K., Ed.; CRC Press: Boca Raton, FL, USA, 2015; pp. 131-132.

5. Bruen, K.; Davidson, K.; Sydes, D.F.E.; Siemens, P.M. Benefits of UV-curable coatings. Eur. Coat. J. 2004, 4, 42.

6. Improving Process Heating System Performance: A Sourcebook for Industry. Available online: http://www. esru.strath.ac.uk/EandE/Sitevisits/EERE_Improving_Proc_Heating_Systems_Performance.pdf (accessed on 21 April 2017).

7. Neral, B.; Šostar-Turk, S.; Vončina, B. Properties of UV-cured pigment prints on textile fabric. Dyes Pigment. 2006, 68, 143-150. [CrossRef]

8. Ferrero, F.; Periolatto, M.; Ferrario, S. Sustainable antimicrobial finishing of cotton fabrics by chitosan UV-grafting: From laboratory experiments to semi industrial scaleup. J. Clean. Prod. 2015, 96, 244-252. [CrossRef]

9. Yuan, H.; Xing, W.; Zhang, P.; Song, L.; Hu, Y. Functionalization of cotton with UV-cured flame retardant coatings. Ind. Eng. Chem. Res. 2012, 51, 5394-5401. [CrossRef]

10. Carosio, F.; Alongi, J. Few durable layers suppress cotton combustion due to the joint combination of layer by layer assembly and UV-curing. RSC Adv. 2015, 5, 71482-71490. [CrossRef]

11. Chen, W.-H.; Chen, P.-C.; Wang, S.-C.; Yeh, J.-T.; Huang, C.-Y.; Chen, K.-N. UV-curable PDMS-containing PU system for hydrophobic textile surface treatment. J. Polym. Res. 2009, 16, 601-610. [CrossRef]

12. Ferrero, F.; Periolatto, M.; Sangermano, M.; Bianchetto, S.M. Water-repellent finishing of cotton fabrics by ultraviolet curing. J. Appl. Polym. Sci. 2008, 107, 810-818. [CrossRef]

13. Periolatto, M.; Basit, A.; Ferri, A.; Bongiovanni, R. Wettability and comfort of cellulosic materials modified by photo grafting of non-fluorinated oligomers. Cellulose 2016, 23, 1447-1458.

14. Xue, C.-H.; Jia, S.-T.; Zhang, J.; Ma, J.-Z. Large-area fabrication of superhydrophobic surfaces for practical applications: An overview. Sci. Technol. Adv. Mat. 2010, 11, 1-15. [CrossRef] [PubMed]

15. Kasturiya, N.; Bhargava, G.S. Liquid repellency and durability assessment: A quick technique. J. Ind. Text. 2003, 32, 187-222. [CrossRef] 
16. Mohsin, M.; Sarwar, N.; Ahmad, S.; Rasheed, A.; Ahmad, F.; Afzal, A.; Zafar, S. Maleic acid crosslinking of C-6 fluorocarbon as oil and water repellent finish on cellulosic fabrics. J. Clean. Prod. 2016, 112, 3525-3530. [CrossRef]

17. Castelvetro, V.; Francini, G.; Ciardelli, G.; Ceccato, M. Evaluating fluorinated acrilic latices as textile water and oil repellent finishes. Text. Res. J. 2001, 71, 399-406. [CrossRef]

18. Shao, H.; Sun, J.Y.; Meng, W.-D.; Qing, F.-L. Water and oil repellent and durable press finishes for cotton based on a perfluoroalkyl-containing multi-epoxy compound and citric acid. Text. Res. J. 2004, 74, 851-855. [CrossRef]

19. Lee, H.J.; Michielsen, S. Preparation of a superhydrophobic rough surface. J. Polym. Sci. Part B Polym. Phys. 2007, 45, 253-261. [CrossRef]

20. Li, Z.-R.; Fu, K.-J.; Wang, L.-J.; Liu, F. Synthesis of a novel perfluorinated acrylate copolymer containing hydroxyethyl sulfone as crosslinking group and its application on cotton fabrics. J. Mater. Process. Technol. 2008, 205, 243-248. [CrossRef]

21. AATCC/ASTM Test Method TS-018-Procedure for Absorbency; American Association of Textile Chemists and Colorists: Research Triangle Park, NC, USA, 2014.

22. AATCC TM173-2015-Calculation of Small Color Difference for Acceptability; American Association of Textile Chemists and Colorists: Research Triangle Park, NC, USA, 2015.

23. Roe, B.; Zhang, X. Durable hydrophobic textile fabric finishing using silica nanoparticles and mixed silanes. Text. Res. J. 2009, 79, 1115-1122. [CrossRef]

(C) 2017 by the authors. Licensee MDPI, Basel, Switzerland. This article is an open access article distributed under the terms and conditions of the Creative Commons Attribution (CC BY) license (http:/ / creativecommons.org/licenses/by/4.0/). 